\title{
Coil Embolization of Pulmonary Arteries as a Palliative Treatment of Diffuse Type I Hepatopulmonary Syndrome
}

\author{
Kimberley Grady MD, Srinath Gowda MD, Pascal Kingah MD, and Ayman O Soubani MD
}

\begin{abstract}
Hepatopulmonary syndrome is a serious complication of liver disease. Type I hepatopulmonary syndrome is associated with diffuse dilatation of the pulmonary vasculature, leading to severe hypoxemia. Liver transplantation is the treatment of choice for this condition. There are limited options for those who are not candidates for liver transplantation. We present the case of a patient who presented with severe hypoxemia requiring $\mathrm{F}_{\mathrm{IO}_{2}}$ of 0.95 with $\mathrm{P}_{\mathrm{aO}_{2}}$ of $59 \mathrm{~mm} \mathrm{Hg}$. Workup revealed $33 \%$ intrapulmonary right-to-left shunt. A pulmonary angiogram showed diffuse dilatation of the pulmonary arteries, especially in the lower lobes. The patient was diagnosed with type I hepatopulmonary syndrome. He was not a candidate for liver transplantation. The patient underwent sequential coil embolizations of the lower lobe pulmonary arteries. He was discharged home on $2 \mathrm{~L}$ of supplemental oxygen. This case demonstrates that coil embolization of dilated pulmonary arteries is a potential palliative treatment for patients with diffuse type I hepatopulmonary syndrome. Key words: hepatopulmonary syndrome; coil embolization; liver transplantation; hypoxemia; right-to-left shunt; liver disease. [Respir Care 2015;60(2):e20-e25. (C) 2015 Daedalus Enterprises]
\end{abstract}

\section{Introduction}

Hepatopulmonary syndrome is an important cause of dyspnea and hypoxemia in patients with liver disease. It results from altered gas exchange due to abnormal capillary dilatation and/or arteriovenous fistulae within the pulmonary vasculature. Hepatopulmonary syndrome is defined by 3 diagnostic criteria: (1) liver disease (most commonly, portal hypertension) with or without cirrhosis, (2) hypoxemia $\left(\mathrm{P}_{\mathrm{aO}_{2}}\right.$ of $<70 \mathrm{~mm} \mathrm{Hg}$ or alveolar-arterial oxygen difference of $>20 \mathrm{~mm} \mathrm{Hg}$ on ambient air), and (3) intrapulmonary vascular dilatations (supported by contrastenhanced echocardiography or abnormal uptake with ra-

Drs Grady, Kingah, and Soubani are affiliated with the Division of Pulmonary \& Critical Care and Sleep Medicine, and Dr Gowda is affiliated with the Division of Pediatric Cardiology, Wayne State University School of Medicine, Detroit, Michigan.

The authors have disclosed no conflicts of interest.

Correspondence: Ayman O Soubani MD, Division of Pulmonary \& Critical Care and Sleep Medicine, Harper University Hospital, Wayne State University School of Medicine, 3990 John R, 3-Hudson, Detroit, MI 48201. E-mail: asoubani@med.wayne.edu.

DOI: $10.4187 /$ respcare 03198 dioactive lung perfusion scan). ${ }^{1}$ Hepatopulmonary syndrome can be further characterized as type I or II (Table 1). Type I hepatopulmonary syndrome is defined as precapillary pulmonary artery dilatation without the presence of arteriovenous fistulas. In contrast, type II hepatopulmonary syndrome is defined as discrete areas of pulmonary arteriovenous fistulas. Type I hepatopulmonary syndrome usually has limited response to $100 \%$ oxygen once there are diffuse vascular changes. ${ }^{2,3}$ The severity of hepatopulmonary syndrome is based on the level of arterial deoxygenation, with severe hepatopulmonary syndrome characterized as $\mathrm{P}_{\mathrm{aO}} \geq 60$ to $<80 \mathrm{~mm} \mathrm{Hg}$ on room air. ${ }^{2}$ There is no association between the severity of hepatopulmonary syndrome and the severity (defined by the Model for EndStage Liver Disease score) or type of liver disease. ${ }^{4}$ The treatment of choice for hepatopulmonary syndrome is liver transplantation. ${ }^{5}$ We present an interesting case of coil embolization as a palliative treatment for a patient with type I hepatopulmonary syndrome who was not a candidate for liver transplantation.

\section{Case Report}

A 59-y-old African-American male with history of COPD, alcohol abuse, hepatitis $\mathrm{C}$, schizophrenia, and depression presented with progressive hypoxia requiring sup- 


\section{Coil Embolization in Diffuse Type I Hepatopulmonary Syndrome}

Table 1. Summary of Type I Versus Type II Hepatopulmonary Syndrome

\begin{tabular}{lll}
\hline \hline & \multicolumn{1}{c}{ Type I Hepatopulmonary Syndrome } & Type II Hepatopulmonary Syndrome \\
\hline $\begin{array}{l}\text { Prevalence } \\
\begin{array}{l}\text { Pulmonary vascular dilatation (based on CT } \\
\text { angiogram) }\end{array}\end{array}$ & $\begin{array}{l}\text { Rare } \\
\text { Diffuse with either normal or spongy appearance }\end{array}$ & $\begin{array}{l}\text { Extremely rare } \\
\text { Discrete areas }\end{array}$ \\
$\begin{array}{l}\text { Arteriovenous fistulas } \\
\text { Mechanism of hypoxemia }\end{array}$ & Not present & Present \\
Response to oxygen & Ventilation/perfusion mismatching, diffusion & True intrapulmonary right-to-left shunt \\
Treatment & limitation, intrapulmonary right-to-left shunt & Poor \\
& Limited, poor once diffuse involvement occurs & Liver transplantation, coil embolization \\
CT = computed tomography & Liver transplantation & \\
\hline
\end{tabular}

plemental oxygen over the previous year. Upon presentation, he had no symptoms suggestive of infection or cardiac decompensation. He had a history of 1.5 pints of liquor/d and a remote history of intravenous drug use. Family history was positive only for pulmonary embolism in his sister. Prior to hospitalization, he was taking albuterol, ipratropium, and budesonide/formoterol fumarate for management of COPD and risperidone and sertraline for management of schizophrenia and depression.

Upon initial physical examination, the patient was afebrile, with a temperature of $36.4^{\circ} \mathrm{C}$, a blood pressure of $137 / 66 \mathrm{~mm} \mathrm{Hg}$, a heart rate of 73 beats/min, a breathing frequency of 34 breaths/min, and $\mathrm{S}_{\mathrm{pO}_{2}}$ of $90 \%$ on $\mathrm{F}_{\mathrm{IO}_{2}}$ of 0.95 via a high-flow nasal cannula. Respiratory and cardiovascular examination was unremarkable. He had hepatomegaly and gynecomastia with no other stigmata of liver disease (no spider nevi, palmar erythema, anicteric sclera, or ascites). The patient had signs of clubbing of fingernails with no evidence of cyanosis. Platypnea and orthodeoxia were also present.

Initial arterial blood gas on $\mathrm{F}_{\mathrm{IO}_{2}}$ of 0.95 via a high-flow (50 L/min) nasal cannula revealed $\mathrm{P}_{\mathrm{aO}_{2}}$ of $59 \mathrm{~mm} \mathrm{Hg}$ with a shunt fraction of $33 \%$. No arterial blood gas was recorded on room air secondary to his initial level of hypoxia. There was concern for intrapulmonary versus intracardiac shunt; therefore, a transthoracic echocardiogram with bubble study was performed. The echocardiogram revealed severe globally depressed left ventricular systolic function with moderately increased left ventricular cavity size. The estimated left ventricular ejection fraction was $20-25 \%$ (an echocardiogram 3 months earlier indicated an ejection fraction of 55\%). The electrocardiogram and cardiac enzymes were within normal limits. The left and right atrial size was normal, as was the right ventricular size and systolic function. The bubble study indicated a persistent foramen ovale with right-to-left shunt. The persistent foramen ovale was felt to be hemodynamically insignificant given the small amount of bubbles that appeared in the left atrium and the normal right atrial size. It was not clear why the patient's left ventricular ejection fraction was depressed on admission; therefore, a repeat echocardiogram was done $2 \mathrm{~d}$ later, and the estimated left ventricular ejection fraction was $55-60 \%$. A dynamic nuclear perfusion scan was done in an attempt to identify intrapulmonary shunt. Results indicated no obvious pulmonary vascular shunt. However, whole body imaging showed significant right-to-left shunting from the lungs to the rest of the body, including uptake in the brain and kidneys, with a measured shunt fraction of $31 \%$. Right heart catheterization indicated that the right ventricular pressure was $28 / 4 \mathrm{~mm} \mathrm{Hg}$, the pulmonary arterial pressure was $17 / 14 \mathrm{~mm} \mathrm{Hg}$ with mean of $15 \mathrm{~mm} \mathrm{Hg}$, the pulmonary capillary wedge pressure was $13 \mathrm{~mm} \mathrm{Hg}$, the cardiac output was $6.7 \mathrm{~L} / \mathrm{min}$, and the cardiac index was $3.22 \mathrm{~L} / \mathrm{min} / \mathrm{m}^{2}$.

Computed tomography of the thorax with a pulmonary embolism protocol revealed no pulmonary emboli or evidence of pulmonary parenchymal disease; however, there was evidence of atelectasis in the right lower lobe, and the pulmonary vasculature was diffusely dilated, especially in the right lower lobe, as shown in Figure 1.

Pulmonary function tests from 3 months prior to hospitalization (obtained from outside hospital records) indicated $\mathrm{FEV}_{1}$ at $81 \%$ of predicted $(2.42 \mathrm{~L}), \mathrm{FVC}$ at $93 \%$ of predicted ( $3.55 \mathrm{~L}), \mathrm{FEV}_{1} / \mathrm{FVC}$ of 0.68 , total lung capacity at $86 \%$ of predicted, and diffusion capacity for carbon monoxide at $36 \%$ of predicted.

There was a suspected connection between his liver disease and hypoxia; therefore, his liver disease was investigation. Liver biopsy indicated evidence of steatosis and cirrhosis. Synthetic liver function was abnormal, with albumin at $2.5 \mathrm{~g} / \mathrm{dL}$ and an international normalized ratio of 1.12. Aspartate aminotransferase, alanine aminotransferase, and alkaline phosphatase were all within normal limits (147, 71, and $99 \mathrm{U} / \mathrm{L}$, respectively). A hepatitis panel was positive for hepatitis C, with a viral load of 3,889,442 IU/mL. The Model for End-Stage Liver Disease score was calculated to be 11 . Portal venous pressures were not measured.

The patient underwent a second right heart catheterization with a pulmonary angiogram. Right-side heart pres- 


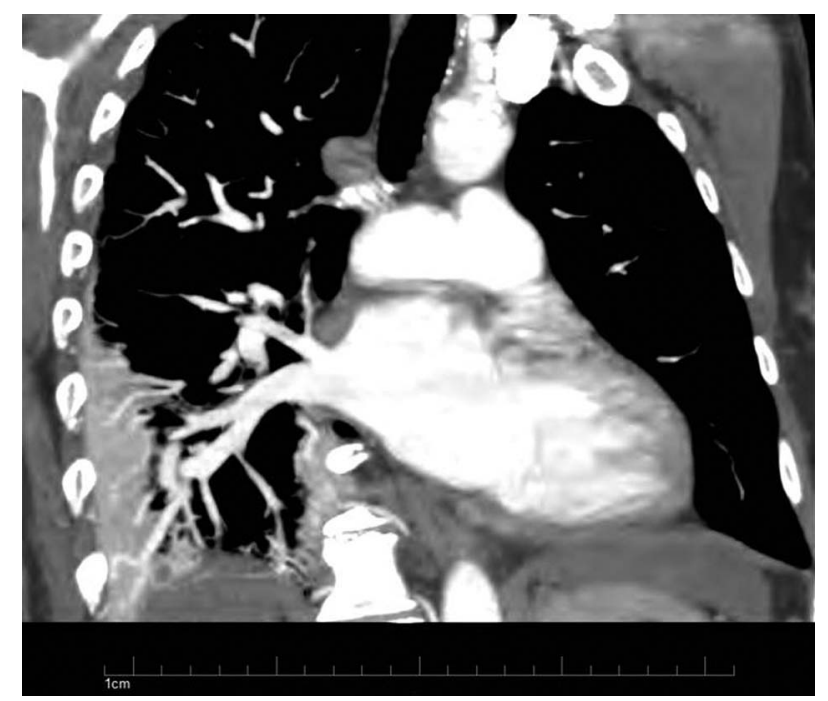

Fig. 1. Initial computed tomography showing dilated pulmonary vasculature, especially in the right lower lobe. There is evidence of atelectasis in the right lower lobe.

sures were within normal range and similar to those of the first right heart catheterization. A pulmonary angiogram showed diffuse pulmonary arterial and venous dilatations with predominance in the bilateral lower lobes (Fig. 2). The right and left pulmonary arteries were severely dilated and measured 30 and $25 \mathrm{~mm}$, respectively. An angiogram of the right and left lower pulmonary arteries indicated severely dilated branches. The distal branches had a tortuous course to the periphery of the lung, and a brisk return of contrast to the dilated right and left lower pulmonary veins was noted. The proximal right and left lower lobe pulmonary arterial branches measured 18 and $20 \mathrm{~mm}$, respectively. There was prominent branching of the right and left upper pulmonary arteries with normal branching patterns. The pulmonary venous return in the right and left upper pulmonary veins was not as brisk as the bilateral lower segments. Significant bubbles were noted in the left atrium after injection into the right and left lower pulmonary arteries. Minimal bubbles were present in the left atrium after injection into the right and left upper pulmonary arteries. Venous blood gases were measured in the upper and lower branches of the bilateral pulmonary veins (Table 2). The $\mathrm{S}_{\mathrm{pO}_{2}}$ on a $2 \mathrm{~L} / \mathrm{min}$ nasal cannula in the right and left upper pulmonary veins was $95 \%$ and $96 \%$, respectively. The $\mathrm{S}_{\mathrm{pO}_{2}}$ on a $2 \mathrm{~L} / \mathrm{min}$ nasal cannula in the right and left lower pulmonary veins was $80 \%$ and $77 \%$, respectively. The combination of significant desaturations in the bilateral lower pulmonary veins, the strongly positive bubble study in the bilateral lower pulmonary arteries, and the severely dilated bilateral lower pulmonary arteries and veins represented diffuse bilateral lower precapillary pulmonary arterial dilatations. In addition, the combination of near-normal venous saturations in the bilateral up-
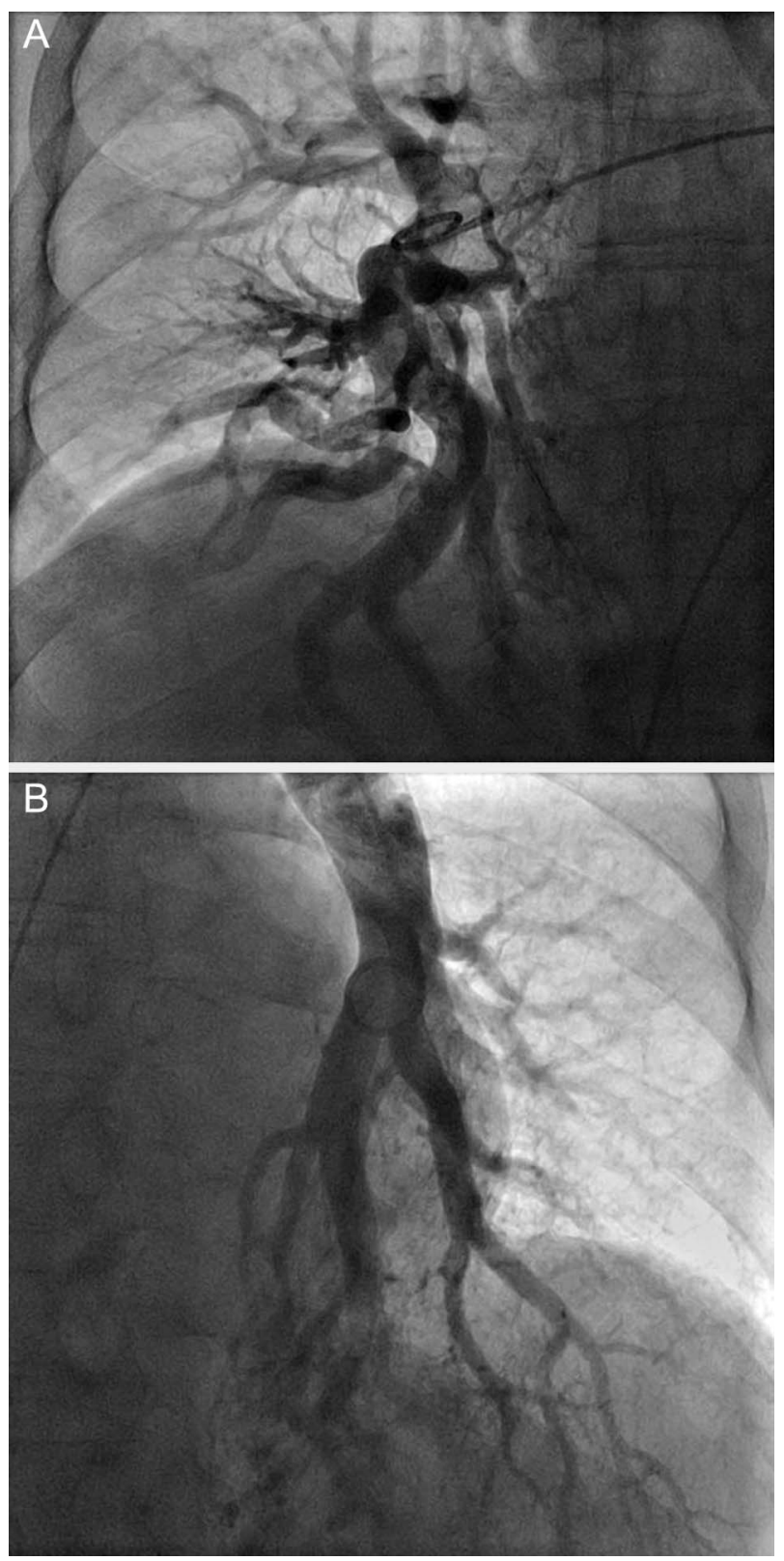

Fig. 2. Pulmonary angiography of the right (A) and left (B) lungs showing diffusely dilated pulmonary arteries in both the right and left lower lobes, respectively.

per pulmonary veins, the weakly positive bubble study in the bilateral upper pulmonary arteries, and the prominent bilateral upper lobe vessels represented mild disease in the bilateral upper pulmonary arteries and veins. The lower oxygen requirements during angiography were probably due to less shunting and ventilation/perfusion mismatching in the lower lobes as the patient assumed the supine position compared with when he was upright (less orthodeoxia).

The patient was diagnosed with type I hepatopulmonary syndrome. Our patient was evaluated by the gastroenter- 


\section{Coil Embolization in Diffuse Type I Hepatopulmonary Syndrome}

Table 2. Blood Gases Measured During Pulmonary Angiography

\begin{tabular}{|c|c|c|c|c|c|}
\hline & $\mathrm{pH}$ & $\mathrm{P}_{\mathrm{CO}_{2}}(\mathrm{~mm} \mathrm{Hg})$ & $\mathrm{P}_{\mathrm{O}_{2}}(\mathrm{~mm} \mathrm{Hg})$ & $\mathrm{HCO}_{3}{ }^{-}(\mathrm{mmol} / \mathrm{L})$ & $\mathrm{S}_{\mathrm{pO}_{2}}(\%)$ \\
\hline Right femoral artery & 7.45 & 31 & 44 & 21 & 82 \\
\hline PV left upper lobe & 7.49 & 28 & 73 & 21 & 96 \\
\hline PV left lower lobe & 7.46 & 32 & 46 & 23 & 77 \\
\hline PV right upper lobe & 7.46 & 29 & 71 & 21 & 95 \\
\hline PV right lower lobe & 7.43 & 34 & 42 & 23 & 80 \\
\hline
\end{tabular}

ologist and determined not to be a candidate for liver transplantation because he had active alcohol intake prior to hospitalization, severe psychiatric problems, and lack of social support. He was also not considered to be a candidate for in-patient treatment of hepatitis $\mathrm{C}$ with interferon because of previous adverse reactions to the medication or with ribavirin because he may not have been able to tolerate the ribavirin-induced anemia given his level of hypoxemia.

Because the patient was deemed unsuitable for liver transplantation and because of his severe persistent hypoxemia, the decision was made to perform sequential coil embolization of the bilateral lower pulmonary arteries. The procedure was planned to be done in 2 sessions because of the extensive pulmonary arterial dilatation and the concern that embolization of a large pulmonary vascular area may be associated with complications such as hypoxemia, pulmonary infarction, or sudden increase in pulmonary arterial pressure. While breathing oxygen at $2 \mathrm{~L} / \mathrm{min}$ via a nasal cannula, the patient underwent temporary balloon occlusion of the right and left lower pulmonary arteries, which resulted in improvement of $\mathrm{S}_{\mathrm{pO}_{2}}$ from $80 \%$ to $89 \%$ on the right and from $77 \%$ to $90-92 \%$ on the left. Coil embolizations of multiple areas of pulmonary arterial dilatations of the right lower lobe were completed. Multiple coils $(5 \times 7 \mathrm{~mm}$ and $8 \times 3 \mathrm{~mm})$ were deployed in multiple areas of right pulmonary arterial dilatations. Post-procedure, his oxygen saturations improved to $88-90 \%$ with 2-3 L/min of supplemental oxygen via a nasal cannula. Eight days after his first procedure, the patient returned to the catheterization lab and underwent coil embolizations of multiple areas of pulmonary arterial dilations of the left lower lobe. Four coils were deployed in segments 8 and 9 of the left lung, and 2 Amplatzer vascular plugs ( 8 and $10 \mathrm{~mm}$; St Jude Medical, St Paul, Minnesota) were placed to better occlude the areas. During this procedure, a repeat angiogram was completed after placement of the coils and vascular plugs. Residual flow through the right pulmonary arterial dilations was found; therefore, 2 Amplatzer vascular plugs (10 and $12 \mathrm{~mm}$ ) were placed proximal to the previous coils. A chest radiograph post-procedure showed

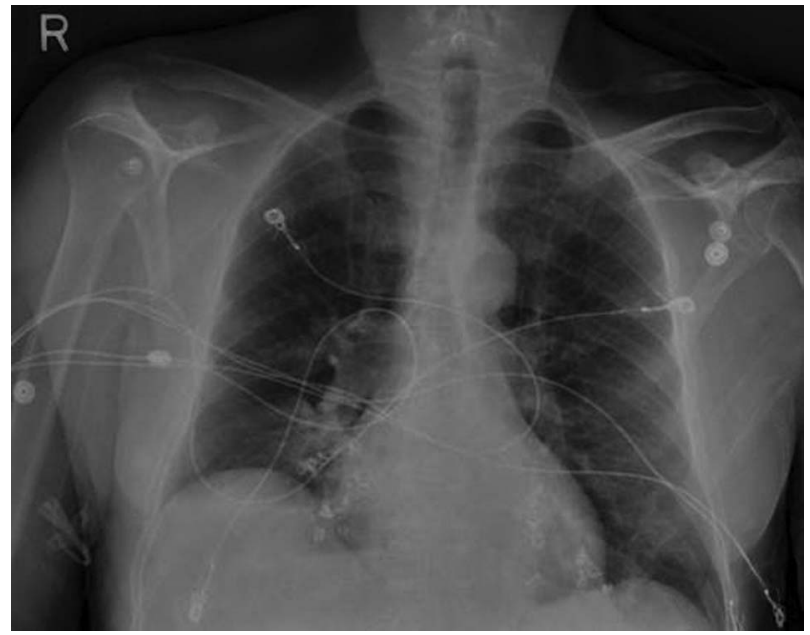

Fig. 3. Post-embolization chest radiograph indicating coil embolization in the bilateral lower lung fields.

multiple vascular coils in the lower lobes with no parenchymal changes (Fig. 3).

The patient was slowly weaned from high-flow oxygen to $2 \mathrm{~L} / \mathrm{min}$ via a nasal cannula. The patient was doing well with stable oxygen requirements 5 months following coil embolization.

\section{Discussion}

Approximately $10-20 \%$ of cirrhotic patients evaluated for liver transplantation are diagnosed with hepatopulmonary syndrome. ${ }^{3}$ It is important to note that there is no association between the severity of hepatopulmonary syndrome and the severity (defined by the Model for EndStage Liver Disease score) or type of liver disease. ${ }^{3,4}$ Also, one study concluded that the abnormality in arterial oxygenation can worsen despite clinically stable liver disease. ${ }^{6}$

The main mechanisms of hypoxemia in patients with type I hepatopulmonary syndrome are related to pulmonary vascular dilatations at the precapillary level that lead to ventilation/perfusion mismatching, a barrier to gas diffusion, and right-to-left shunting. Ventilation/perfusion 
mismatching occurs when there is an overperfusion of the alveolar capillary bed, especially in the less ventilated dependent lung zones. This is exacerbated by the impaired hypoxic pulmonary vasoconstriction seen in these patients. Dilatation of the pulmonary microvessels at the alveolar level increases the distance oxygen molecules must traverse to bind to red blood hemoglobin in the center of the alveolar capillaries, creating a functional diffusion barrier to oxygen exchange. ${ }^{7}$ This is exacerbated by the decreased transit time commonly seen in patients with elevated cardiac output associated with cirrhosis. Patients may also have true anatomic shunting in the form of direct arteriovenous communications. ${ }^{2,3,8-10}$ The pathogenesis of intrapulmonary vascular dilatation is controversial; however, many theories are supported by the increased presence of vasoactive substances such as nitric oxide in patients with hepatopulmonary syndrome..$^{1,2,8}$ Some propose that there is enhanced pulmonary production of nitric oxide likely secondary to an increased presence of endothelial nitric oxide synthase and inducible nitric oxide synthase in the pulmonary vasculature. ${ }^{2,3,8}$ In the setting of cirrhotic patients, bacterial translocation increases tumor necrosis factor alpha, which causes alveolar macrophages to produce inducible nitric oxide synthase within the pulmonary microvasculature, thus increasing nitric oxide. ${ }^{2,3,8}$ Others propose that a diseased liver inadequately metabolizes pulmonary vasoactive substances such as nitric oxide. ${ }^{2,8}$ Endothelin-1 activation of endothelin B receptors is known to cause nitric oxide-mediated vasodilatation of endothelial cells. Endothelin B receptors are increased in the pulmonary (not systemic) circulation of patients with cirrhosis and portal hypertension. ${ }^{10}$ Increased pulmonary angiogenesis has also been suggested in a rat model of hepatopulmonary syndrome. ${ }^{11}$

Pharmacologic therapy has a limited role in the management of hepatopulmonary syndrome. Agents such as pentoxifylline (a nonspecific phosphodiesterase inhibitor that blocks the synthesis of tumor necrosis factor alpha), methylene blue (an inhibitor of guanylate cyclase), and endothelin B receptor blockers have been tried in a small number of studies with variable results. ${ }^{12-15}$ The treatment of choice for hepatopulmonary syndrome is liver transplantation, with a 5-y survival rate after liver transplantation of $76 \% .{ }^{16}$ In contrast, the 5-y survival of patients who do not undergo liver transplantation is $23 \%$, with a median survival of 24 months. In the setting of hepatic-pulmonary vascular disorders, orthotropic liver transplantation is indicated when $\mathrm{P}_{\mathrm{aO}}$ is $\geq 50$ to $<60 \mathrm{~mm} \mathrm{Hg.}{ }^{8}$ However, baseline $\mathrm{P}_{\mathrm{aO}}$, as in our patient, is associated with worse survival irrespective of the decision to transplant. ${ }^{15}$ In one of the largest studies on the effects of liver transplantation on hepatopulmonary syndrome, there was sustained, statistically significant improvement in $\mathrm{P}_{\mathrm{aO}_{2}}$ after 12 months following liver transplantation. ${ }^{16}$ The mean pre-liver trans- plant $\mathrm{P}_{\mathrm{aO}}$ was $57 \mathrm{~mm} \mathrm{Hg}$, and the mean post-liver transplant $\mathrm{P}_{\mathrm{aO}_{2}}$ was $87 \mathrm{~mm} \mathrm{Hg}(P=.001)$.

Our patient was not a candidate for liver transplantation given his active alcohol abuse prior to hospitalization, severe psychiatric problems, and lack of social support. However, he had a constant requirement for $\mathrm{F}_{\mathrm{IO}_{2}}$ of 0.85-0.95 via a high-flow nasal cannula. Coil embolization was considered to be a palliative treatment, with significant improvement in hypoxemia. Coil embolization has been used for the treatment of type II hepatopulmonary syndrome; however, is not commonly used for type I hepatopulmonary syndrome. ${ }^{17,18}$ There is a case report of a patient with type I hepatopulmonary syndrome awaiting liver transplantation who underwent multiple coil embolization of pulmonary arterial dilatations. ${ }^{19}$ However, in that case, a pulmonary angiogram indicated pulmonary arterial dilations only in the right lower lobe and, less predominantly, in the right upper lobe. The patient was discharged home on oxygen supplementation maintaining $\mathrm{S}_{\mathrm{pO}_{2}}$ of $.95 \%$ while awaiting liver transplantation. The long-term effect of the coil embolization in that case was not reported. ${ }^{19}$

There are few published case reports that demonstrate the effects of coil embolization in patients with diffuse type I hepatopulmonary syndrome ${ }^{16-18}$; however, there are data regarding the effects in patients with other disorders of the pulmonary vasculature. Contrast echocardiogram after coil embolization of pulmonary arteriovenous malformations has demonstrated significant reduction of the pulmonary shunt. ${ }^{20}$ In a study of patients with underlying hereditary hemorrhagic telangiectasia who underwent coil embolization for pulmonary arteriovenous malformations, there was a significant increase in arterial oxygen saturation $(P=.004) .{ }^{21}$ The study also indicated that coil embolization did not significantly increase pulmonary artery pressure in patients with pre-existing mild-to-moderate pulmonary hypertension, and no patients developed right heart failure. Other side effects include pleurisy in $10-15 \%$ of cases, pulmonary infarction, retrograde pulmonary embolism, sepsis, and malposition of coils or vascular plugs..$^{20,22}$ Recanalization and collateralization of pulmonary arteriovenous malformations post-embolization are common reasons for procedure failure and occur in $\sim 5-19 \%$ of cases. ${ }^{4,22}$ Therefore, close follow-up of patients who undergo coil embolization as a palliative treatment for hepatopulmonary syndrome is probably necessary. Computed tomography pulmonary angiogram is an important tool for monitoring the success and control of vascular malformations post-coil embolization. ${ }^{22}$

In our patient, the combination of refractory hypoxia and a pulmonary angiogram indicating diffuse pulmonary vascular dilatations with predominance in the bilateral lower lobes supports the diagnosis of diffuse type I hepatopulmonary syndrome. The patient was discharged home on $2 \mathrm{~L} / \mathrm{min}$ of supplemental oxygen after multiple coil 


\section{Coil Embolization in Diffuse Type I Hepatopulmonary Syndrome}

embolizations of pulmonary arterial dilatations. This case suggests that patients may benefit from coil embolizations of the pulmonary arterial dilatations as a palliative treatment for diffuse type I hepatopulmonary syndrome.

\section{REFERENCES}

1. Rodríguez-Roisin R, Krowka MJ. Hepatopulmonary syndrome-a liver-induced lung vascular disorder. N Engl J Med 2008;358(22): 2378-2387.

2. Rodríguez-Roisin R, Krowka MJ, Hervé P, Fallon MB, ERS Task Force. Pulmonary-hepatic vascular disorders (PHD). Eur Respir J 2004;24(5):861-880.

3. Sawant P, Vashishtha C, Nasa M. Management of cardiopulmonary complications of cirrhosis. Int J Hepatol 2011;2011:280569.

4. Cartin-Ceba R, Swanson KL, Krowka MJ. Pulmonary arteriovenous malformations. Chest 2013;144(3):1033-1044.

5. Moorman J, Saad M, Kosseifi S. Hepatitis C virus and the lung: implications for therapy. Chest 2005;128(4):2882-2892.

6. Krowka MJ, Dickson ER, Cortese DA. Hepatopulmonary syndrome. Clinical observations and lack of therapeutic response to somatostatin analogue. Chest 1993;104(2):515-521.

7. Katsuta Y, Honma H, Zhang XJ, Ohsuga M, Komeichi H, Shimizu $\mathrm{S}$, et al. Pulmonary blood transit time and impaired arterial oxygenation in patients with chronic liver disease. J Gastroenterol 2005; 40(1):57-63

8. Ho V. Current concepts in the management of hepatopulmonary syndrome. Vasc Health Risk Manag 2008;4(5):1035-1041.

9. Fritz JS, Fallon MB, Kawut SM. Pulmonary vascular complications of liver disease. Am J Respir Crit Care Med 2013;187(2):133-143.

10. Grace JA, Angus PW. Hepatopulmonary syndrome: update on recent advances in pathophysiology, investigation, and treatment. J Gastroenterol Hepatol 2013;28(2):213-219.
11. Zhang J, Luo B, Tang L, Wang Y, Stockard CR, Kadish I, et al Pulmonary angiogenesis in a rat model of hepatopulmonary syndrome. Gastroenterology 2009;136(3):1070-1080.

12. Tanikella R, Philips GM, Faulk DK, Kawut SM, Fallon MB. Pilot study of pentoxifylline in hepatopulmonary syndrome. Liver Transpl 2008;14(8):1199-1203.

13. Zhang J, Ling Y. Pentoxifylline attenuation of experimental hepatopulmonary syndrome. J Appl Physiol 2007;102(3):949-955.

14. Schenk P, Madl C, Rezaie-Majd S, Lehr S, Müller C. Methylene blue improves the hepatopulmonary syndrome. Ann Intern Med 2000; 133(9):701-706.

15. Zhang J, Ling Y. Attenuation of experimental hepatopulmonary syndrome in endothelin B receptor-deficient rats. Am J Physiol Gastrointest Liver Physiol 2009;296(4):G704-G708.

16. Swanson KL, Wiesner RH, Krowka MJ. Natural history of hepatopulmonary syndrome: impact of liver transplantation. Hepatology 2005;41(5):1122-1129.

17. Lee HW, Suh KS, Kim J, Shin WY, Yi NJ, Jae HJ, et al. Pulmonary artery embolotherapy in a patient with type I hepatopulmonary syndrome after liver transplantation. Korean J Radiol 2010;11(4):485489.

18. Saad NE, Lee DE, Waldman DL, Saad WE. Pulmonary arterial coil embolization for the management of persistent type I hepatopulmonary syndrome after liver transplantation. J Vasc Interv Radiol 2007; 18(12):1576-1580.

19. Ryu JK, Oh JH. Hepatopulmonary syndrome: angiography and therapeutic embolization. Clin Imaging 2003;27(2):97-100.

20. Andersen PE, Kjelsen AD. Interventional treatment of pulmonary arteriovenous malformations. World J Radiol 2010;2(9):339-344.

21. Shovlin CL, Tighe HC, Davies RJ, Gibbs JS, Jackson JE. Embolisation of pulmonary arteriovenous malformations: no consistent effect on pulmonary artery pressure. Eur Respir J 2008;32(1):162-169.

22. Lee EW, Grant JD, Loh CT, Kee ST. Bronchial and pulmonary arterial and venous interventions. Semin Respir Crit Care Med 2008; 29(4):395-404 